\title{
Capacity of entanglement in local operators
}

\author{
Pratik Nandy \\ Centre for High Energy Physics, Indian Institute of Science, \\ C.V. Raman Avenue, Bangalore-560012, India \\ E-mail: pratiknandy@iisc.ac.in
}

ABSTRACT: We study the time evolution of the excess value of capacity of entanglement between a locally excited state and ground state in free, massless fermionic theory and free Yang-Mills theory in four spacetime dimensions. Capacity has non-trivial time evolution and is sensitive to the partial entanglement structure, and shows a universal peak at early times. We define a quantity, the normalized "Page time", which measures the timescale when capacity reaches its peak. This quantity turns out to be a characteristic property of the inserted operator. This firmly establishes capacity as a valuable measure of entanglement structure of an operator, especially at early times similar in spirit to the Rényi entropies at late times. Interestingly, the time evolution of capacity closely resembles its evolution in microcanonical and canonical ensemble of the replica wormhole model in the context of the black hole information paradox.

KEywords: Conformal Field Theory, Field Theories in Higher Dimensions, Field Theories in Lower Dimensions

ArXiv EPrint: 2106.00228 


\section{Contents}

1 Introduction 1

2 A brief review on capacity of entanglement 3

$\begin{array}{lll}3 & \text { Setup } & 4\end{array}$

4 Free, massless fermionic theory in 4-dimensions $\quad 6$

$\begin{array}{lll}4.1 & \text { Quasi-particle entanglement at late times } & 8\end{array}$

$\begin{array}{lr}4.2 \text { Capacity for charged fermionic operator } & 9\end{array}$

$\begin{array}{lll}4.3 & \text { Spin dependence and capacity } & 10\end{array}$

4.4 Operator insertion and "Page time" 11

5 Free Yang-Mills theory in 4-dimensions 13

$\begin{array}{lll}5.1 & \text { Late time behavior and EPR interpretation } & 15\end{array}$

6 Conclusion and summary $\quad 16$

\section{Introduction}

Over the recent years, entanglement entropy has played a substantial role in understanding many exciting phenomena, ranging from condensed matter systems to gravity. For example, in condensed matter systems, it appears to be a useful tool to characterize topological ordered phases [1-3], whereas entanglement emerges as an indispensable property to understand the AdS/CFT duality $[4,5]$ via the Ryu-Takayanagi (RT) prescription [6, 7]. Initially, it was derived for the static case, and later it was generalized to the time-dependent case [8] and extended by including quantum corrections $[9,10]$. This formulation, which goes by the name of 'Entanglement wedge reconstruction' has turned out to be crucial in the understanding of the black hole information paradox [11, 12] by the newly developed island prescription $^{1}[15,16]$. The motivation is to replace the standard RT surface by Quantum Extremal Surface (QES) [17] which eventually reproduces the Page curve [18, 19].

On a somewhat different note, an information-theoretic quantity, known as the capacity of entanglement [20-23] or capacity in short, recently gained some attention, for example, in the context of the information paradox [24, 25]. It was shown that, like entanglement entropy, capacity could also truly probe the phase transition that happens at the Page time $[24,25]$. It either shows a peak or changes discontinuously between two phases at Page time, similar in spirit to the subregion complexity [26, 27]. Although less familiar, capacity has been studied in condensed matter systems like the Kitaev model, and it appears to be

\footnotetext{
${ }^{1}$ See $[13,14]$ for excellent reviews.
} 
well suited for characterizing topologically ordered states [28]. It is defined via the second derivative of Rényi entropy $S_{A}^{(m)}$ with respect to the replica parameter [20, 24]

$$
C_{E}\left(\rho_{A}\right)=\lim _{m \rightarrow 1} m^{2} \partial_{m}^{2} \log \left[\operatorname{tr}\left(\rho_{A}^{m}\right)\right]=\lim _{m \rightarrow 1} m^{2} \partial_{m}^{2}\left((1-m) S_{A}^{(m)}\right),
$$

where $\rho_{A}$ is the density matrix in subsystem $A$, formed by tracing out the degrees of freedom from the complement subsystem $\bar{A}$ and $m$ is the replica parameter. Recently capacity was studied in random pure states [29]. It is mostly influenced by the partially connected geometries where subleading saddle points contribute. In this fashion, it naturally emerges as a useful quantity to understand the entanglement spectrum.

In this paper, we study the excess value of capacity given by the difference between the capacities in excited state and ground state in free, massless fermionic theory (both charged and uncharged) and free Yang-Mills theory in four spacetime dimensions. The excited states are defined by acting local operators on the ground state. The series of Rényi entropies have been calculated in various cases [30-45]. The main result is that the excess Rényi entropies increase monotonically until their maximum value, and they characterize the given operator's quantum entanglement structure at late times. One may ask if it is possible to capture the entanglement structure at early times by other information-theoretic quantities. Here we show the answer is affirmative, and the excess value of capacity is one of them.

The excess value of capacity shows a peak at a timescale that we referred to as normalized "Page time". This quantity is normalized with respect to the position of the inserted operator. Interestingly, the peak value of capacity turns out to be universal. We call it "Page time" 2 due to the close resemblance with the evolution of capacity in replica geometry [24]. However, we do not have any black holes in our case. The normalized "Page time" turns out to be a characteristic property of the inserted operator. Thus the capacity is an excellent probe to understand the entanglement structure at early times compared to Rényi entropies, which is a suitable probe at late times. Additionally, the crossover happens at the Page time when the entanglement is partial. When the entanglement entropy reaches the maximum, capacity dies off either to zero (for uncharged fermionic and free Yang-Mills case) or constant asymptotic value (for charged fermionic case). This asymptotic value depends on the chemical potential. The late-time behavior of capacity can be interpreted as the generation of EPR pairs. When the EPR pairs take away all the entanglement, capacity vanishes.

Another nice feature is observed in Yang-Mills theory in 4-dimensions. In general, for large- $N$ theory, the entanglement entropy is ill-defined to leading order once we take the $m \rightarrow 1$ limit from Rényi entropy. This is because the subleading terms dominate over the leading term. On the other hand, we find capacity is perfectly well defined on $m \rightarrow 1$ limit and shows the expected behavior. Moreover, capacity turns out to be sensitive to the internal degrees of freedom of the inserted operator.

The paper is structured as follows. In section 2, we review the main aspects of the capacity of entanglement with a two-qubit example. In section 3, we discuss the setup. Section 4 involves the discussion on free, massless fermionic theory in 4-dimensions. We

\footnotetext{
${ }^{2}$ This is just a characteristic time scale, we do not need to take this nomenclature too seriously.
} 
compute the time evolution of the capacity of entanglement for both uncharged and charged fermions and give the possible interpretation from the EPR point of view at late times. We also describe the close similarity with the replica wormhole model. In section 5, we compute the time evolution of capacity in free Yang-Mills theory in 4-dimensions. We conclude with a summary of the main results and future problems in section 6 . Throughout our discussion, we set $c=\hbar=1$.

\section{A brief review on capacity of entanglement}

We first briefly introduce the capacity of entanglement and consider an example [20] where it can be compared with other quantities like entanglement and Rényi entropies. Consider a system that is described by a density matrix $\rho$. We divide the system into two parts, subsystem $A$ and its complement $\bar{A}$. We trace out the degrees of freedom in subsystem $\bar{A}$ to get the reduced density matrix of subsystem $A$ given by

$$
\rho_{A}=\operatorname{tr}_{\bar{A}} \rho .
$$

From this we can compute the Rényi entropy with a single index $m$ as [46]

$$
S_{A}^{(m)}=\frac{1}{1-m} \log \left[\operatorname{tr}\left(\rho_{A}^{m}\right)\right]
$$

for integer $m>1$. One can analytically continue for non-integer $m$. In fact the most important limit is to take $m \rightarrow 1$, which gives the entanglement entropy ${ }^{3}$

$$
S_{A}^{E E}=\lim _{m \rightarrow 1} S_{A}^{(m)}=\lim _{m \rightarrow 1} \frac{1}{1-m} \log \left[\operatorname{tr}\left(\rho_{A}^{m}\right)\right] .
$$

The gravity dual of Rényi entropy was considered, and its derivative (with respect to the index $m$ ) was shown to be dual to the area of cosmic brane with tension proportional to $(m-1)$ [48]. This tension term backreacts in the bulk. In the limit $m \rightarrow 1$, the tension vanishes, the Rényi entropy becomes the entanglement entropy. Alongside, the cosmic brane becomes the minimal surface and does not backreact in the bulk. This minimal surface is dual to boundary subregion and has been studied extensively in the past few years $[6-8]$.

Writing in terms of modular Hamiltonian $\mathcal{K}_{A}=-\log \rho_{A}$, the entanglement entropy becomes the expectation value of the modular Hamiltonian [20]

$$
S_{A}^{E E}\left(\rho_{A}\right)=-\operatorname{tr}\left(\rho_{A} \log \rho_{A}\right)=\operatorname{tr}\left(\rho_{A} \mathcal{K}_{A}\right)=\left\langle\mathcal{K}_{A}\right\rangle,
$$

where subscript $A$ indicates that we are taking the expectation value in subsystem $A$.

The capacity of entanglement (or capacity in short) is another information-theoretic quantity that has gained some interest recently. As an information-theoretic quantity, it measures the variance of the modular Hamiltonian ${ }^{4}[20]$

$$
C_{E}\left(\rho_{A}\right)=\operatorname{tr}\left[\rho_{A}\left(-\log \rho_{A}\right)^{2}\right]-\left[-\operatorname{tr}\left(\rho_{A} \log \rho_{A}\right)\right]^{2}=\left\langle\mathcal{K}_{A}^{2}\right\rangle-\left\langle\mathcal{K}_{A}\right\rangle^{2} .
$$

\footnotetext{
${ }^{3}$ An alternate prescription of obtaining entanglement entropy from Rényi entropy is given in [47].

${ }^{4}$ More generally, one can define capacity in terms of the relative entropy variance between two density matrices. When one of the density matrix becomes maximally mixed, i.e., proportional to the identity, the relative entropy variance becomes the capacity [23].
} 
In general, the Rényi entropies capture the full entanglement spectrum of the density matrix. The entanglement entropy and capacity are two characteristic features of this entanglement spectrum. In terms of Rényi entropy the capacity is given by [20]

$$
C_{E}\left(\rho_{A}\right)=\lim _{m \rightarrow 1} m^{2} \partial_{m}^{2} \log \left[\operatorname{tr}\left(\rho_{A}^{m}\right)\right]=\lim _{m \rightarrow 1} m^{2} \partial_{m}^{2}\left((1-m) S_{A}^{(m)}\right) .
$$

In general, the capacity shows very different behavior than entanglement or Rényi entropies, although the capacity and entanglement entropy might be equal in some cases [20]. An interesting behavior of capacity was observed in the context of information paradox [24]. In the end of the world (EoW) brane model [49] the capacity exhibits a peak at Page time and indicates a phase transition between a fully disconnected and a fully connected geometry of replica wormholes. ${ }^{5}$ In the moving mirror model [51], capacity jumps discontinuously at Page time. In both cases, the entanglement entropy increases monotonically and saturates to the black hole entropy. This indicates capacity could be an indicator of phase transition between two phases.

As an example we will study a two-qubit system to understand the capacity, entanglement and Rényi entropies. Let us consider the state [20]

$$
|\psi\rangle=\cos (\beta / 2)|01\rangle+e^{i \chi} \sin (\beta / 2)|10\rangle,
$$

with $0 \leq \beta \leq \pi$ and $0 \leq \chi<2 \pi$. If one traces out the second spin, the reduced density matrix becomes $\rho_{1}=\operatorname{diag}(u, 1-u)$ where $u=\cos ^{2}(\beta / 2)$. A direct computation gives

$$
\begin{aligned}
S_{E E} & =-[(1-u) \ln (1-u)+u \ln u], \\
C_{E} & =(1-u)(\ln (1-u))^{2}+u(\ln u)^{2}-[(1-u) \ln (1-u)+u \ln u]^{2}, \\
S_{1}^{(m)} & =\frac{1}{1-m} \log \left[(1-u)^{m}+u^{m}\right] .
\end{aligned}
$$

The variation of entanglement entropy, capacity, and $m=2,3$ Rényi entropies with $u$ is shown in figure 1. The interesting point is that at $u=0$ and $u=1$, the state (2.7) is separable which implies all of the entanglement measures should vanish. On the other hand, at $u=0.5$, the state is maximally entangled (EPR state), which gives entanglement entropy and all Rényi entropies a value of $\log 2$, while the capacity vanishes. The capacity peaks up a value $C_{E}^{\max }=0.4392$ at $u=0.0832$ and $u=0.9168$ which is in fact a partially entangled state (see figure 1). This concludes that while the EPR state is formed the entanglement is carried away by EPR pairs, and thus capacity becomes zero. We will encounter this fact again when we discuss free, massless fermionic and free Yang-Mills theory in the latter part of the paper.

\section{Setup}

Let us consider a local operator in Schödinger picture $\mathcal{W}(-L, \mathbf{q})$ located at $x=-L$ at time $t=-t$. Here $\mathbf{q}=\{y, z\}$ are other spatial directions. The directions $\mathbf{q}$ will not play

\footnotetext{
${ }^{5}$ See also [50] for the Page transition in replica wormhole model considering gravitational bath.
} 


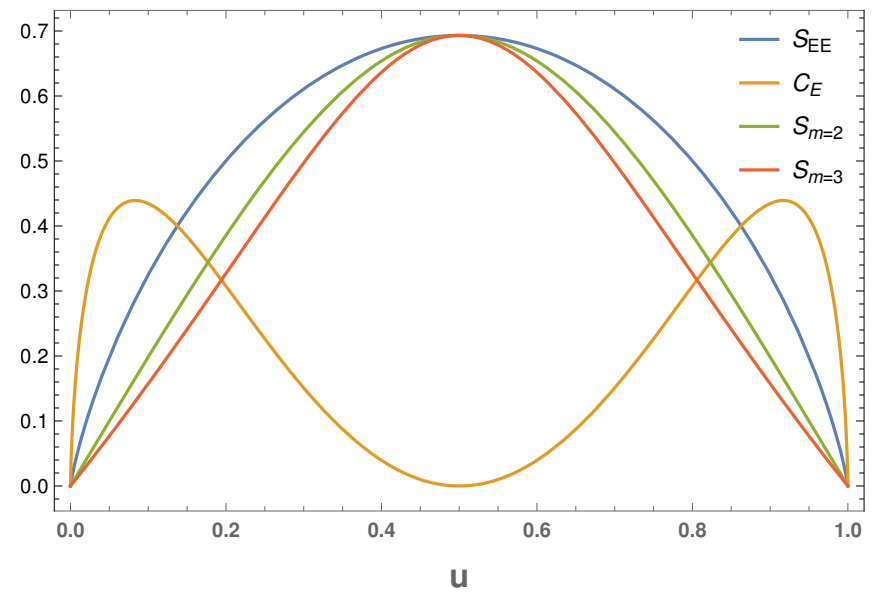

Figure 1. Variation of capacity, entanglement, and Rényi entropies with $u$. Note that the behavior of capacity is very different from the entanglement and Rényi entropies. Rényi and entanglement entropies reach maximum (at maximally entangled state) at $u=0.5$, where the capacity vanishes, while the capacity shows peaks in some intermediate partially entangled state.

any role in our case, and we suppress them often. The operators act on the ground state and create locally excited states of the form [30-32]

$$
\left|\Psi_{\mathcal{W}}\right\rangle=N e^{-i \mathcal{H} t} e^{-\epsilon \mathcal{H}} \mathcal{W}(-L, \mathbf{q})|0\rangle
$$

where $\epsilon$ is introduced by requiring a finite norm of the state. One usually defines the operator in the Heisenberg picture as $\mathcal{W}(\tau,-L, \mathbf{q})=e^{i \mathcal{H} \tau} e^{-\epsilon \mathcal{H}} \mathcal{W}(-L, \mathbf{q})$ with complex time $\tau=-\epsilon-i t$ treating them as real parameters. The density matrix is calculated as

$$
\rho_{\mathcal{W}}=\left|\Psi_{\mathcal{W}}\right\rangle\left\langle\Psi_{\mathcal{W}}\left|=N^{2} \mathcal{W}\left(\tau_{e},-L, \mathbf{q}\right)\right| 0\right\rangle\langle 0| \mathcal{W}^{\dagger}\left(\tau_{l},-L, \mathbf{q}\right),
$$

where $\tau_{e}=-\epsilon-i t$ and $\tau_{l}=\epsilon-i t$. The goal is to calculate Rényi entropies in Euclidean time and finally do the analytic continuation.

Now we consider region $A$ as half of the spacetime, i.e., $x \geq 0$. The reduced density matrix in the region $A$ is $\rho_{A}^{e}=\operatorname{tr}_{\bar{A}} \rho^{e}$, where $e$ stands for the excited state and $\bar{A}$ is the region $x<0$. Similarly, one can consider the ground state $|0\rangle$ and trace out the region $\bar{A}$ to get the density matrix $\rho_{A}^{g}=\operatorname{tr}_{\bar{A}}|0\rangle\langle 0|$. Here $g$ stands for the ground state. The Rényi entropies and capacity are given by

$$
S_{A}^{(m), e / g}=\frac{1}{1-m} \log \left[\operatorname{tr}\left(\rho_{A}^{e / g}\right)^{m}\right], \quad C_{E}^{e / g}=\lim _{m \rightarrow 1} m^{2} \partial_{m}^{2}\left[(1-m) S_{A}^{(m), e / g}\right] .
$$

Hence the increased value of Rényi entropy is given by subtracting the ground state value from that of the excited state

$$
\Delta S_{A}^{(m)}=\frac{1}{1-m} \log \left[\frac{\operatorname{tr}\left(\rho_{A}^{e}\right)^{m}}{\operatorname{tr}\left(\rho_{A}^{g}\right)^{m}}\right] .
$$

As shown in [31, 32], the Rényi entropy can be calculated using the replica trick $[46,52,53]$. We first evaluate the $2 m$-point correlation function on full $m$-sheeted manifold $\Xi_{m}$ and the 


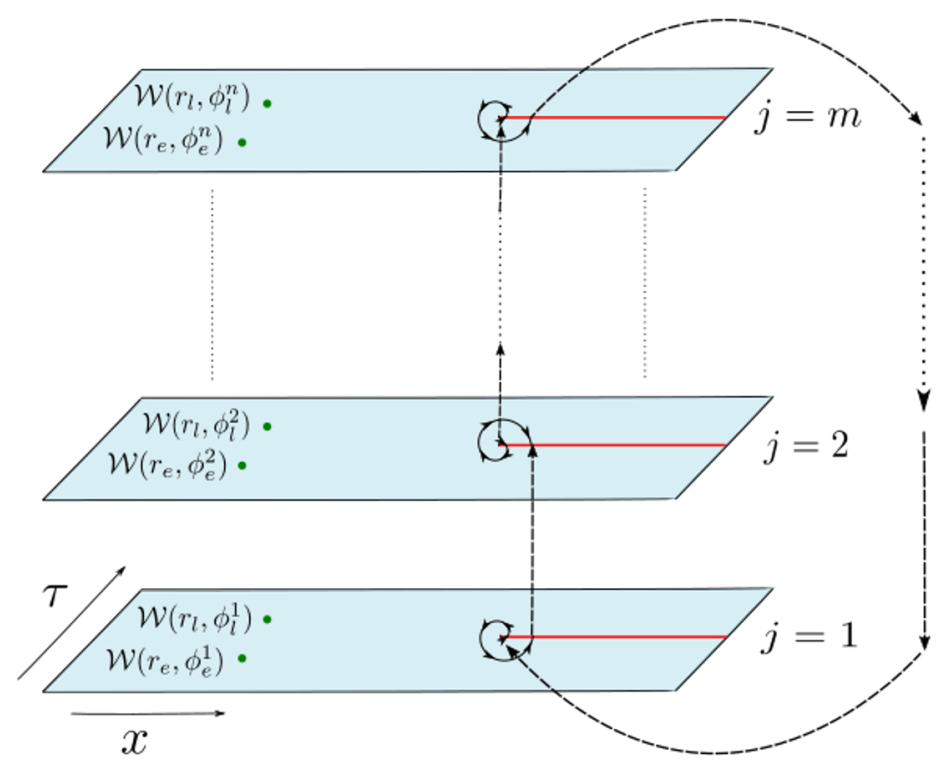

Figure 2. Evaluation of $\Delta S_{A}^{(m)}$ using the replica trick.

2-point correlation function on single sheeted manifold $\Xi_{1}$ and then take the ratio between them (see figure 2). The result is [32]

$$
\Delta S_{A}^{(m)}=\frac{1}{1-m} \log \left[\frac{\left\langle\mathcal{W}^{\dagger}\left(r_{l}, \phi_{l}^{m}\right) \mathcal{W}\left(r_{e}, \phi_{e}^{m}\right) \cdots \mathcal{W}^{\dagger}\left(r_{l}, \phi_{l}^{1}\right) \mathcal{W}\left(r_{e}, \phi_{e}^{1}\right)\right\rangle_{\Xi_{m}}}{\left\langle\mathcal{W}^{\dagger}\left(r_{l}, \phi_{l}^{1}\right) \mathcal{W}\left(r_{e}, \phi_{e}^{1}\right)\right\rangle_{\Xi_{1}}^{m}}\right],
$$

where the polar coordinates $(r, \phi)$ on $(\tau, x)$ plane are defined as $\phi_{e, l}^{j}=\phi_{e, l}^{1}+2 \pi(j-1)$ with $0<\phi<2 \pi m$ and $j=1, \cdots, m$. The excess of capacity is now straightforwardly defined as

$$
\Delta C_{E}=\lim _{m \rightarrow 1} m^{2} \partial_{m}^{2}\left((1-m) \Delta S_{A}^{(m)}\right) .
$$

In later sections, we will calculate the excess capacity ${ }^{6}$ in free fermionic and free Yang-Mills theory.

\section{Free, massless fermionic theory in 4-dimensions}

Our first field-theoretic example is to consider free, massless fermions in 4-dimensions. We consider states that are obtained acted by a fermionic operator $\Psi_{a}$ on the ground state. The Rényi entropies are given by [32]

$\Delta S_{A}^{(m)}=\frac{1}{1-m} \log \left[\left(\frac{t+L}{4 t}\right)^{m}\left[\left(\frac{t-L}{t}\right)\left(\gamma^{t} \gamma^{1}\right)_{a a}+2\right]^{m}+\left(\frac{t-L}{4 t}\right)^{m}\left[-\left(\frac{t+L}{t}\right)\left(\gamma^{t} \gamma^{1}\right)_{a a}+2\right]^{m}\right]$

for $t \geq L$, and $\Delta S_{A}^{(m)}=0$ for $t<L$. Here $\gamma^{t}$ and $\gamma^{1}$ are the elements of Dirac's $\gamma^{\mu_{-}}$ matrices. Their explicit forms are not important to our discussion, but one can look at [32]

\footnotetext{
${ }^{6}$ Abusing the terminology, we sometimes refer to this as to capacity only.
} 


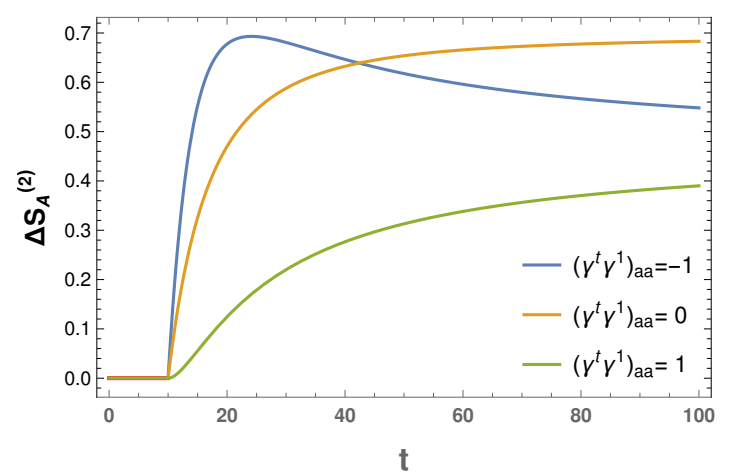

(a) $\Delta S_{A}^{(2)}$ at early times.

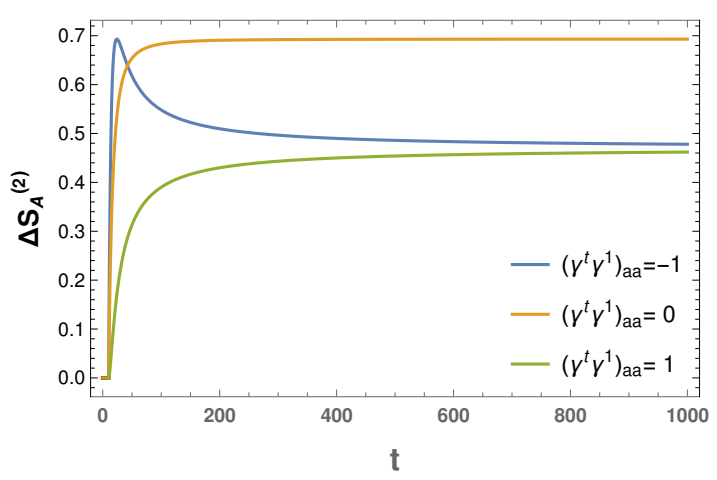

(b) $\Delta S_{A}^{(2)}$ at late times.

Figure 3. Time evolution of Rényi entropy $\Delta S_{A}^{(2)}$ with various choices of $\left(\gamma^{t} \gamma^{1}\right)_{a a}=0, \pm 1$. Note that for $\left(\gamma^{t} \gamma^{1}\right)_{a a}=0$, the Rényi entropy approaches to $\log 2$ as time evolves. This is due to the generation of EPR pairs. For $\left(\gamma^{t} \gamma^{1}\right)_{a a}= \pm 1$ it approaches a constant value $(\approx 0.47)$ at late times. Here we choose $L=10$.

for details. The range of the elements of the Hermitian matrix $\gamma^{t} \gamma^{1}$ is $-1 \leq \gamma \leq 1$, where $\gamma \equiv\left(\gamma^{t} \gamma^{1}\right)_{a a}$ for brevity. In particular, we will consider values of $\gamma=0, \pm 1$. Note that the elements are real. Explicitly this combination tells about the spin direction, which we will describe later. Once we take the late time limit, i.e., $t \gg L$ we get [32]

$$
\Delta S_{A}^{(m) f}=\frac{1}{1-m} \log \left[\left(\frac{2+\gamma}{4}\right)^{m}+\left(\frac{2-\gamma}{4}\right)^{m}\right],
$$

where " $f$ " stands for final value (at late times). It is easy to see for $\gamma=0$, the Rényi entropies become $\Delta S_{A}^{(m) f}=\log 2$ for all $m$ (see figure 3 ). For $\gamma= \pm 1$, the late time entropy is $\Delta S_{A}^{(m) f}=[1 /(1-m)] \log \left[\left(3^{m}+1\right) / 4^{m}\right]$ which approaches to $\log (4 / 3) \approx 0.2877$ for $m \rightarrow \infty$, which is considered as the lower bound of Rényi entropies. Note that for the cases we consider here, the regulator $\epsilon$ will not appear in the expression of entropies and capacity as we take the $\epsilon \rightarrow 0$ limit. This contrasts with the $2 d$ holographic CFTs, where the entropies are not saturated at late times, but they diverge as $\log (t / \epsilon)$.

One can now proceed to calculate the capacity of entanglement using eq. (3.6 $)^{7}$

$$
\Delta C_{E}=\frac{\left(t^{2}-L^{2}\right)\left[(2 t-L \gamma)^{2}-\gamma^{2} t^{2}\right]}{16 t^{4}}\left(\log \left[\frac{(2 t-L \gamma+\gamma t)(t+L)}{(2 t-L \gamma-\gamma t)(t-L)}\right]\right)^{2}, \quad t \geq L
$$

Of course, for $t<L$, capacity vanishes. On the other hand, at late times $(t \gg L)$ it approaches to

$$
\Delta C_{E}^{f}=\frac{\left(4-\gamma^{2}\right)}{16}\left[\log \left(\frac{2+\gamma}{2-\gamma}\right)\right]^{2}
$$

\footnotetext{
${ }^{7}$ Note that the entropy and capacity will depend on the representation of $\gamma$ matrices, and we are choosing a basis where $\gamma^{t} \gamma^{1}=\operatorname{diag}(1,-1,1,-1)$. Here we are considering a physical situation where the anti-particles have a different probability of moving in left and right directions. When $\left(\gamma^{t} \gamma^{1}\right)_{a a}=0$, the probability will be the same to move in both directions. See [32] for more details.
} 


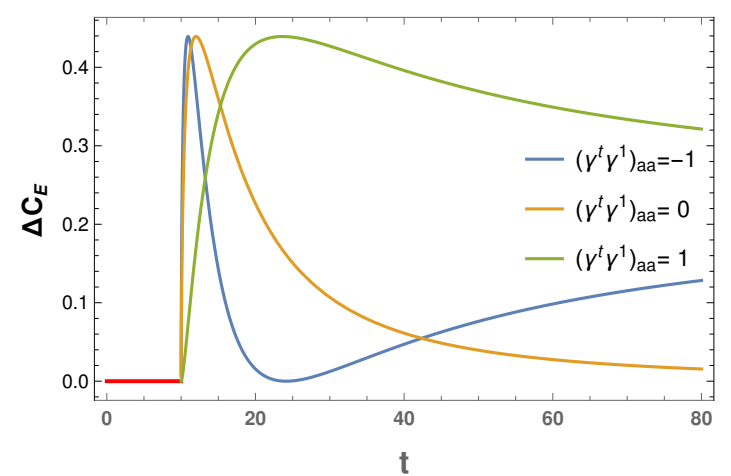

(a) $\Delta C_{E}$ at early times.

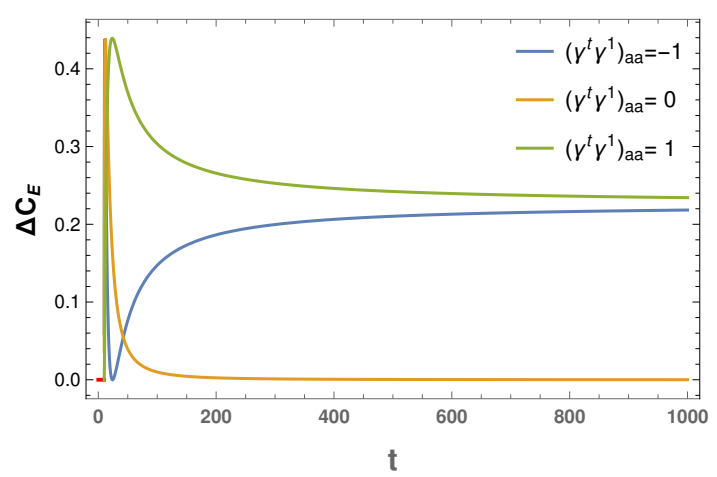

(b) $\Delta C_{E}$ at late times.

Figure 4. Time evolution of excess capacity $\Delta C_{E}$ with various choices of $\left(\gamma^{t} \gamma^{1}\right)_{a a}=0, \pm 1$. Note that for $\left(\gamma^{t} \gamma^{1}\right)_{a a}=0$, capacity approaches zero as time evolves. This is attributed to the formation of EPR pairs. For $\left(\gamma^{t} \gamma^{1}\right)_{a a}= \pm 1$ it approaches a constant value $(\approx 0.2263)$ at late times. The maxima for the capacity occurs at $t=10.9293,11.9968,23.6386$ for $\gamma=-1,0,1$ respectively. For all the cases, the maximum value of capacity is 0.4392 . Here we choose $L=10$, although the maximum value does not depend on $L$. The red line corresponds to $t<L$.

It is easy to see for $\gamma=0$, the late-time capacity vanishes, while for $\gamma= \pm 1$, the capacity approaches a constant value $3(\log 3)^{2} / 16 \approx 0.226303$, which can be anticipated from figure 4. We will again derive the above equation (4.4) from the EPR interpretation. One crucial difference between capacity and Rényi/entanglement entropies is that capacity always shows a peak at early times for any $\gamma$. These peaks in capacity have close similarity with the EoW brane model [24], where it demands a phase transition between disconnected and connected phase at Page time. We will come back to this point again. Also, note that for $\gamma= \pm 1$, Rényi entropies take different values for different $m$ at late times while the capacity takes a universal peak value and depends only on the inserted operator. The peak happens at a partially entangled state, and hence the operators can be characterized by the time where capacity shows the maximum universal value. This implies capacity could, in principle, give enough information of the inserted operator, especially at early times.

\subsection{Quasi-particle entanglement at late times}

At late times the value of excess capacity $\Delta C_{E}$ has a natural interpretation in terms of quasi-particle entanglement between EPR pairs. One splits the local operator into leftmoving and right-moving modes as [32]

$$
\Psi_{a}=\Psi_{a}^{L \dagger}+\Psi_{a}^{R \dagger}+\Phi_{a}^{L}+\Phi_{a}^{R}
$$

while the vacuum $|0\rangle=|0\rangle_{L} \otimes|0\rangle_{R}$ is defined as $(X=L, R), \Psi_{a}^{X}|0\rangle=\Phi_{a}^{X}|0\rangle=0$. Here the momentum modes have non-trivial anti-commutation, which depends on $\gamma$, the spindirection [32]. In our case, we define a locally excited state by the action of the operator $\Psi_{a}$ on vacuum

$$
|\Omega\rangle=N \Psi_{a}|0\rangle,
$$


where $N$ is the normalization. After decomposing $\Psi_{a}$ in terms of momentum modes (4.5) and normalizing we get [32]

$$
|\Omega\rangle=\frac{1}{\sqrt{2}}\left[\Psi_{a}^{L \dagger}|0\rangle_{L} \otimes|0\rangle_{R}+|0\rangle_{L} \otimes \Psi_{a}^{R \dagger}|0\rangle_{R}\right] .
$$

One can directly compute the reduced density matrix for region $A$ by tracing out the left-moving modes

$$
\rho_{A}=\operatorname{tr}_{L}|\Omega\rangle\langle\Omega|=\frac{1}{4}\left[(2+\gamma)|0\rangle_{R}\left\langle\left. 0\right|_{R}+(2-\gamma) \mid \Psi_{a}^{R}\right\rangle\left\langle\left.\Psi_{a}^{R}\right|_{R}\right]\right.
$$

where $\gamma=\left(\gamma^{t} \gamma^{1}\right)_{a a}$ as defined before and $\left|\Psi_{a}^{R}\right\rangle=(1-\gamma / 2)^{-1 / 2} \Psi_{a}^{R \dagger}|0\rangle_{R}$. The capacity can be calculated by first calculating the Rényi entropy and then taking $m \rightarrow 1$ limit, after which we get

$$
\Delta C_{E}=\frac{\left(4-\gamma^{2}\right)}{16}\left[\log \left(\frac{2+\gamma}{2-\gamma}\right)\right]^{2}
$$

which is the same as (4.4). The final value of $\Delta C_{E}$ has spin dependence, as seen from the expression. When $\gamma=0$, capacity vanishes. This is because for $\gamma=0$, the reduced density matrix (4.8) becomes a maximally entangled EPR state. We can see the analogy from the 2-qubit model. Comparing eq. (4.8) and eq. (2.7) in terms of density matrices, we can write

$$
u=\frac{2+\gamma}{4}
$$

where $u=\cos ^{2}(\beta / 2)$ (see eq. (2.7)). For $\gamma=0$, it readily gives $u=1 / 2$, and hence the reduced density matrix becomes $\rho_{1}=\operatorname{diag}(1 / 2,1 / 2)$, a maximally entangled state, for which capacity vanishes. Thus, our late-time interpretation of capacity is consistent with the 2-qubit model. For $\gamma= \pm 1$, the entanglement is partial. Thus, capacity never vanishes, which again matches our intuition developed in section 2 .

\subsection{Capacity for charged fermionic operator}

We now discuss the charged fermionic operator case [36]. If the theory admits a global symmetry, one can define a charge. The charged Rényi entropy was introduced holographically [54, 55], and later it was calculated in field theory. To illustrate the charged Rényi entropy, we first need to define the charged density matrix [36]

$$
\rho_{A}^{c}=\frac{e^{\mu q_{A}} \rho_{A}}{\operatorname{tr}_{A} e^{\mu q_{A}} \rho_{A}},
$$

where $\rho_{A}$ is the density matrix without charge, $q_{A}$ is the global charge, and $\mu$ is the chemical potential which can be either real or purely imaginary. The charged Rényi entropies and charged capacity are defined with respect to this charged density matrix

$$
S_{A}^{(m), c}=\frac{1}{1-m} \log \left[\operatorname{tr}\left(\rho_{A}^{c}\right)^{m}\right], \quad C_{E}^{c}=\lim _{m \rightarrow 1} m^{2} \partial_{m}^{2}\left((1-m) S_{A}^{(m), c}\right) .
$$


The Rényi entropy can be calculated similarly to the uncharged case, but with some subtle caveats. See [36] for the detailed calculation. In either case, we define the excess amount of charged capacity through the excess amount of charged Rényi entropy as

$$
\Delta C_{E}^{c}=\lim _{m \rightarrow 1} m^{2} \partial_{m}^{2}\left((1-m) \Delta S_{A}^{(m), c}\right) .
$$

The calculation gives the charged Rényi entropy as [36]

$$
\Delta S_{A}^{(m), c}=\frac{1}{1-m} \log \left[\frac{(t+L)^{m}\left(2+\gamma \frac{t-L}{t}\right)^{m}+(t-L)^{m}\left(2-\gamma \frac{t+L}{t}\right)^{m} e^{-2 \pi m \mu}}{\left[2\left\{(t-L) e^{-2 \pi \mu}+(t+L)\right\}-\gamma\left(e^{-2 \pi \mu}-1\right) \frac{t^{2}-L^{2}}{t}\right]^{m}}\right], \quad t \geq L
$$

and $\Delta S_{A}^{(m), c}=0$ for $t<L$. The capacity is calculated as

$$
\Delta C_{E}^{c}=\frac{\left(t^{2}-L^{2}\right)\left[(2 t-L \gamma)^{2}-\gamma^{2} t^{2}\right]}{4\left(2 t^{2} \cosh \pi \mu+\left(\gamma t^{2}+2 L t-\gamma L^{2}\right) \sinh \pi \mu\right)^{2}}\left(2 \pi \mu+\log \left[\frac{(2 t-L \gamma+\gamma t)(t+L)}{(2 t-L \gamma-\gamma t)(t-L)}\right]\right)^{2},
$$

valid for $t \geq L$ while it vanishes for $t<L$. The late time behavior is

$$
\Delta C_{E}^{c, f}=\frac{\left(4-\gamma^{2}\right)}{4(2 \cosh \pi \mu+\gamma \sinh \pi \mu)^{2}}\left[2 \pi \mu+\log \left(\frac{2+\gamma}{2-\gamma}\right)\right]^{2} .
$$

Note that for $\mu=0$, we get back to eq. (4.3) and eq. (4.4) as desired. The capacity is plotted with various choices of spin direction, $\gamma=0, \pm 1$ (see figure 5a). Primarily we focus on the late-time behavior of entanglement entropy and capacity (for $\gamma=0$ )

$$
\begin{aligned}
\Delta S_{E E}^{c, f} & =\frac{2 \pi \mu}{1+e^{2 \pi \mu}}+\log \left(1+e^{-2 \pi \mu}\right), \\
\Delta C_{E}^{c, f} & =\pi^{2} \mu^{2} \operatorname{sech}^{2} \pi \mu .
\end{aligned}
$$

The variations of excess entanglement entropy and capacity with $\mu$ are shown in figure 5b. We see that capacity again peaks up a value of 0.4392 at $|\mu|=0.3818$ while the entanglement entropy is maximum of $\log 2$ for vanishing potential. Conclusively, the finite value of chemical potential gives a finite contribution to the capacity at late times. We will see this behavior is very similar to the evolution of capacity in the EoW brane model in the canonical ensemble. For large chemical potential, both capacity and entanglement entropy behave similarly. In fact for $|\mu| \rightarrow \infty$, both approaches zero. This is because the corresponding state becomes a product state. Finally, one can have the quasi-particle interpretation at late times for charged case also, and we refer to [36] for further details.

\subsection{Spin dependence and capacity}

We have seen that capacity intrinsically depends on the direction of spin for both the charged and the uncharged cases. As discussed in [32], $\gamma>0$ implies that the entangled particles have more tendency to go towards the left side (away from region $A)^{8}$ of the

\footnotetext{
${ }^{8}$ Note that region $A$ is defined as the region $x \geq 0$.
} 


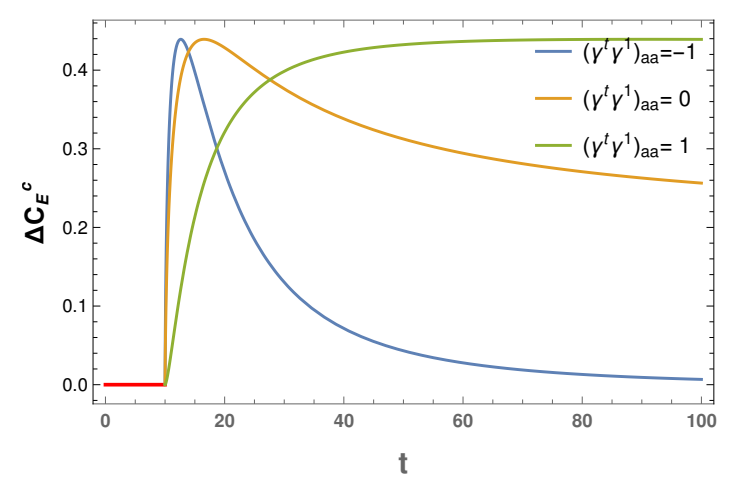

(a) $\Delta C_{E}^{c}$ with time.

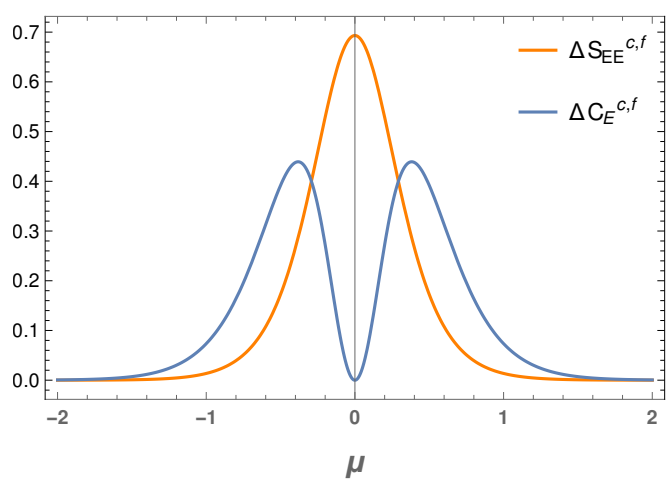

(b) $\Delta S_{E E}^{c, f}$ and $\Delta C_{E}^{c, f}$ with $\mu(\gamma=0)$.

Figure 5. (a) Late-time behavior of charged capacity. Note that for $\gamma=0$, the capacity does not approach to zero, in contrast with uncharged case. We set $\mu=1 / 2 \pi$. (b) Variation of the final value of entanglement entropy $\left(\Delta S_{E E}^{c, f}\right)$ and capacity $\left(\Delta C_{E}^{c, f}\right)$ with chemical potential for $\gamma=0$. Here we choose $L=10$. The red line in (a) corresponds to $t<L$.

insertion point. On the other hand, for $\gamma<0$, particles have more tendency to propagate to the right side (towards region $A$ ) of the insertion point. For $\gamma=0$, particles are equally likely to go both sides. For the uncharged case, we see from figure 4 that capacity always reaches its unique peak value of 0.4392 irrespective of the value of $\gamma$, although the time when it reaches the peak depends on $\gamma$. We see that $\gamma=1$ shows up both maximum and minimum. At late times, capacity reaches a constant value $(\approx 0.2263)$ for $\gamma \pm 1$ while it vanishes for $\gamma=0$, due to the formation of EPR pairs. The time when capacity reaches its peak is truly a characteristic feature of the insterted operator, implying it is a good probe for the entanglement structure.

For the charged case, the time to reach the peak depends on the chemical potential, but the peak value is independent of it. Here for $\gamma=0$, capacity does not vanish at late times. Instead, it reaches an asymptotic value. In contrast to the uncharged case, $\gamma=1$ does not show any minimum. In general, the evolution of capacity is quite different from Rényi or entanglement entropies which usually increase monotonically. Instead, the capacity has peaks and dips, which provide information about partially entangled states at intermediate steps.

\subsection{Operator insertion and "Page time"}

Let us first concentrate on the uncharged case with $\gamma=0$. We call the timescale $t_{P}$ when capacity shows the peak as the "Page time". Note that this is just nomenclature, following the phase transition observed in [24]. To get the Page time we differentiate eq. (4.3) with respect to $t$ (we also set $\gamma=0$ ) and equate it to zero to obtain the nonlinear equation

$$
\frac{1}{2} \log \left(\frac{t+L}{t-L}\right)=\frac{t}{L}
$$

In our case, setting $L=10$, we get the numerical solution of Page time, $t_{P} \approx 11.9968$, for which the peak (maximum) value of capacity is $\Delta C_{E}^{\max }=0.4392$. An exciting fact is that this maximum value is independent of $L$, although the corresponding Page time is 
dependent on $L$. This can be circumvented by noticing that the time always appears as a form of $T=t / L$ in the expression of capacity. Hence, the evolution of capacity with $T$ will be universal, and one can define a quantity

$$
T_{P}=\frac{t_{P}}{L}
$$

which we call normalized "Page time". This means we are normalizing everything with respect to the point where we insert the operator. This quantity defines the timescale when capacity will reach its peak value and a true characteristic property of the inserted operator. In our case, we get $T_{P}=1.19968$. Notice that the maximum value of capacity always reaches 0.4392 irrespective of $L$ and spin direction. This is the exact value of maximum capacity in the 2-qubit model we studied before. It appears as a universal value, whenever the states are maximally entangled (and hence the entanglement entropy reaches $\log 2$ ), the maximum capacity is bound to be 0.4392 .

For the charged fermionic case, to get the "Page time" we differentiate eq. (4.15) and equate it to zero, getting

$$
\frac{1}{2} \log \left(\frac{t+L}{t-L}\right)=\frac{(L-\pi \mu t) \sinh \pi \mu+(t-\pi \mu L) \cosh \pi \mu}{L \cosh \pi \mu+t \sinh \pi \mu},
$$

which reduces to eq. (4.19) for $\mu=0$. Note that the solution of $t$ obtained from eq. (4.21) will depend non-trivially on $\mu$, but surprisingly for fixed $\mu$, the normalized Page time is universal. Hence our conclusion will not change. The (normalized) Page time is an intrinsic property of the operator from the quantum entanglement viewpoint.

Surprisingly, the time evolution of capacity closely resembles the evolution studied in the EoW brane model [24]. Figure 6 and figure 7 show both the evolution of excess entanglement entropy and excess capacity for uncharged and charged cases (we set $\gamma=0$ ). While the entanglement entropy smoothly varies, a crossover happens for capacity. It quickly vanishes as time evolves for the uncharged case. This plot mimics the evolution of capacity in the EoW brane model in the microcanonical ensemble. On the other hand, capacity saturates to an asymptotic value for the charged case, depending on the chemical potential. This resembles the evolution of capacity in the EoW model for the canonical ensemble. The late-time value of capacity suggests that the chemical potential plays a similar role to temperature in the canonical ensemble. ${ }^{9}$ Note that this identification is somewhat naive at this stage, and we do not have any gravity or black holes here. Nevertheless, the resemblance is quite striking and factually lies on the entanglement properties of quasi-particles, especially that the capacity is a probe for partial entanglement and hence acquires maximum contribution from partially connected geometries in the EoW model [29]. It would be interesting to investigate this connection in more detail in the future.

\footnotetext{
${ }^{9}$ From the quasi-particle point of view, the non-zero value of capacity at late times suggests that, instead of maximally entangled EPR pairs, the entanglement is carried away by randomly entangled pairs [20]. Still, the capacity does vanish at $|\mu| \rightarrow \infty$, implying that the chemical potential might control the nature of entanglement in the quasi-particles.
} 


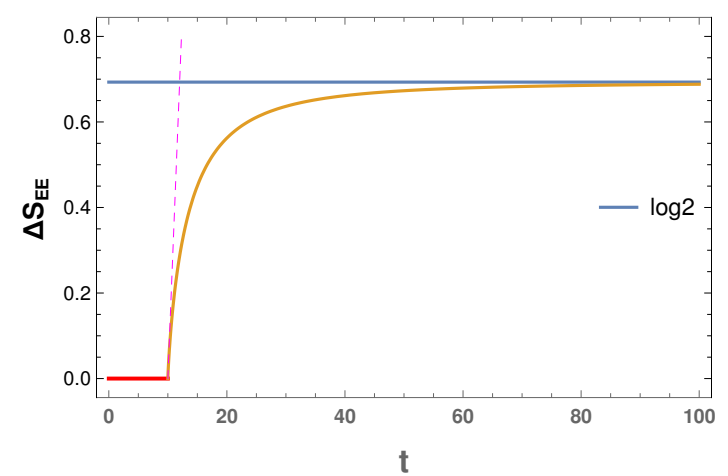

(a) $\Delta S_{E E}$ with time.

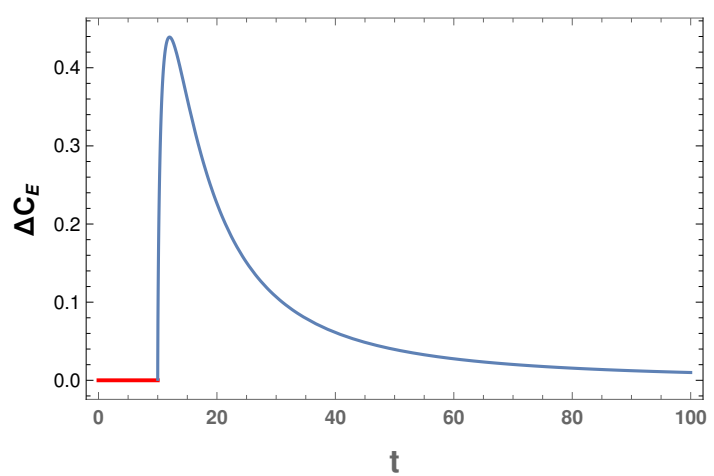

(b) $\Delta C_{E}$ with time.

Figure 6. Plot of (a) entanglement entropy and (b) capacity of entanglement for $\left(\gamma^{t} \gamma^{1}\right)_{a a}=0$ for the uncharged case. Here we choose $L=10$. At $t \approx 11.9968$, capacity reaches a peak where the "Page transition" occurs and dies off late. The entanglement entropy saturates to $\log 2$. These plots are strikingly similar to the entanglement entropy and capacity plots of the EoW brane model in the microcanonical ensemble [24].

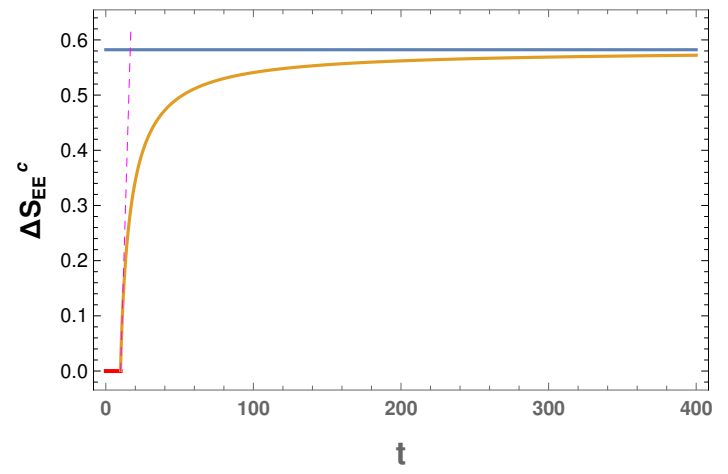

(a) $\Delta S_{E E}^{c}$ with time.

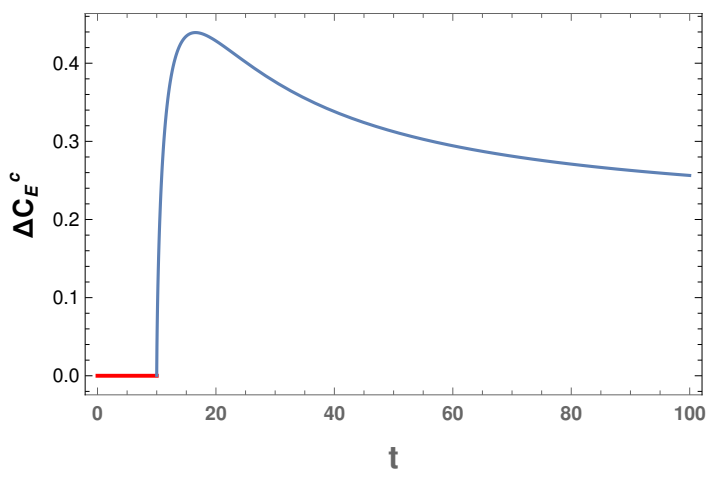

(b) $\Delta C_{E}^{c}$ with time.

Figure 7. Plot of (a) entanglement entropy and (b) capacity of entanglement for $\left(\gamma^{t} \gamma^{1}\right)_{a a}=0$ for the charged case. Here we choose $L=10$ and $\mu=1 / 2 \pi$. At $t \approx 16.5518$, capacity reaches a peak where the "Page transition" occurs. Note that capacity does not vanish at late times. Instead, it reaches a constant value $\Delta C_{E}^{c, f}=0.1996612$ at late times. The late time entanglement entropy saturates to $\Delta S_{E E}^{c, f}=0.5822$. Again these plots closely resemble the EoW brane model in the canonical ensemble [24].

\section{$5 \quad$ Free Yang-Mills theory in 4-dimensions}

Here we briefly discuss the properties of capacity in gauge theory with $\mathrm{U}(N)$ symmetry, especially for the free Yang-Mills theory in four dimensions. We consider the operator of the form

$$
\operatorname{tr}\left[\Phi^{\mathcal{I}}\right]
$$

where $\Phi$ is a real, massless scalar field and $\mathcal{I}$ measures the number of scalar fields. We take it as some $\mathcal{O}(1)$ number. For example, for $\mathcal{I}=1$, the operator is equivalent to the free, 


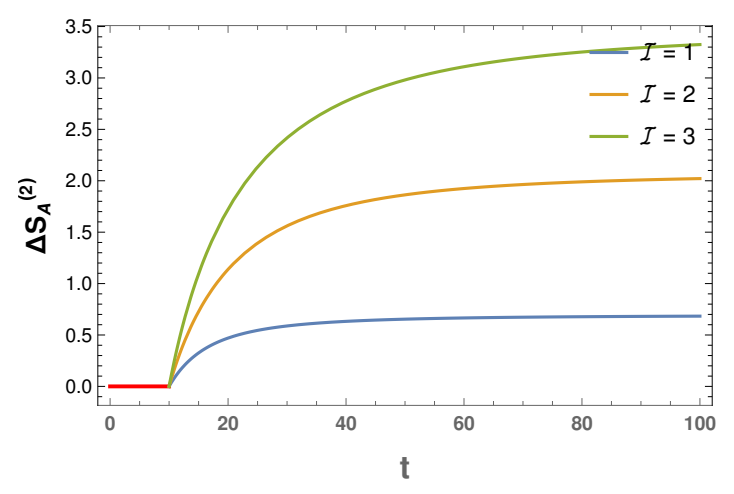

(a) $\Delta S_{A}^{(2)}$ with time.

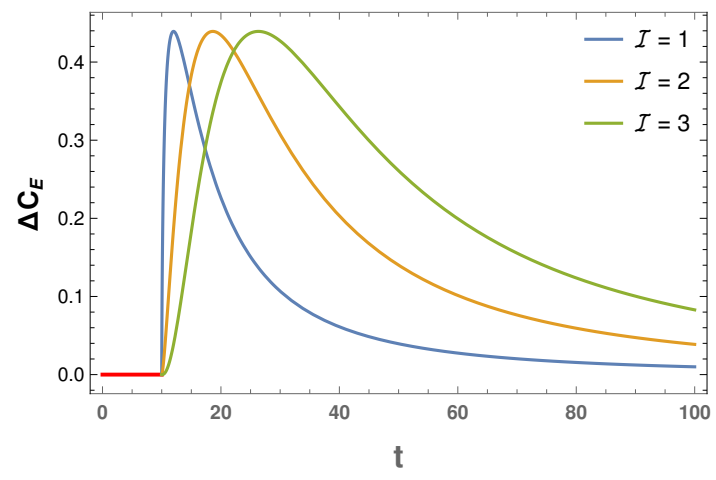

(b) $\Delta C_{E}$ with time.

Figure 8. Plot of (a) $\Delta S_{A}^{(2)}$ and (b) $\Delta C_{E}$ for different values of $\mathcal{I}$. Here we choose $L=10 . \Delta S_{A}^{(2)}$ saturates to $\log 2,3 \log 2$ and $5 \log 2$ for $\mathcal{I}=1,2$ and 3 respectively. On the other hand, $\Delta C_{E}$ peaks up a value 0.4392 for $t=11.9968, t=18.6242$ and $t=26.3257$ for $\mathcal{I}=1,2$ and 3 respectively. Finally it vanishes at late times for all $\mathcal{I}$. The red line corresponds to $t<L$.

massless scalar field operator. It is challenging to calculate Rényi entropy in general, but for large- $N$, the leading behavior of Rényi entropies are given by [34]

$$
\Delta S_{A}^{(m)}=\frac{1}{1-m} \log \left[2^{-\mathcal{I} m}\left\{\left(1-\frac{L}{t}\right)^{\mathcal{I} m}+\left(1+\frac{L}{t}\right)^{\mathcal{I} m}\right\}\right] . \quad t \geq L
$$

From this we can calculate capacity

$$
\Delta C_{E}=\frac{\mathcal{I}^{2}\left(1-\frac{L^{2}}{t^{2}}\right)^{\mathcal{I}}}{\left[\left(1+\frac{L}{t}\right)^{\mathcal{I}}+\left(1-\frac{L}{t}\right)^{\mathcal{I}}\right]^{2}}\left[\log \left(\frac{t+L}{t-L}\right)\right]^{2}, \quad t \geq L
$$

while for $t<L$, the capacity vanishes. At late times, $t \gg L$ we again see that $\Delta C_{E}$ vanishes, irrespective of value of $\mathcal{I}$. On the other hand, at late times, the Rényi entropies depend on both $\mathcal{I}$ and $m, \Delta S_{A}^{(m), f}=(\mathcal{I} m-1) \log 2 /(m-1)$. In the midway the capacity increases and shows a peak at some partially entangled state and goes to zero at late times when the states are maximally entangled (figure 8). But the most important fact is that capacity is well defined when we take $m \rightarrow 1$ limit from Rényi entropy. As observed in [34], we can see that $m \rightarrow 1$ limit breaks down once we try to calculate the entanglement entropy. To get the (normalized) Page time we differentiate eq. (5.3) with respect to $t$, and set it to zero to get

$$
\frac{\mathcal{I}}{2} \log \left[\frac{T_{P}+1}{T_{P}-1}\right]=\frac{\left(T_{P}+1\right)^{\mathcal{I}}+\left(T_{P}-1\right)^{\mathcal{I}}}{\left(T_{P}+1\right)^{\mathcal{I}}-\left(T_{P}-1\right)^{\mathcal{I}}},
$$

where we have written everything in terms of the normalized Page time defined in eq. (4.20). From the numerical solution, we can easily get the normalized Page time as $T_{P}=1.19968$, 1.86242 and 2.63257 for $\mathcal{I}=1,2$ and 3 respectively. See figure 8, where the Page time has been obtained for $L=10$, and it perfectly matches with the normalized Page time. Notice that for $\mathcal{I}=1$, eq. (5.4) reduces to eq. (4.19), which is reflected on the same numerical 


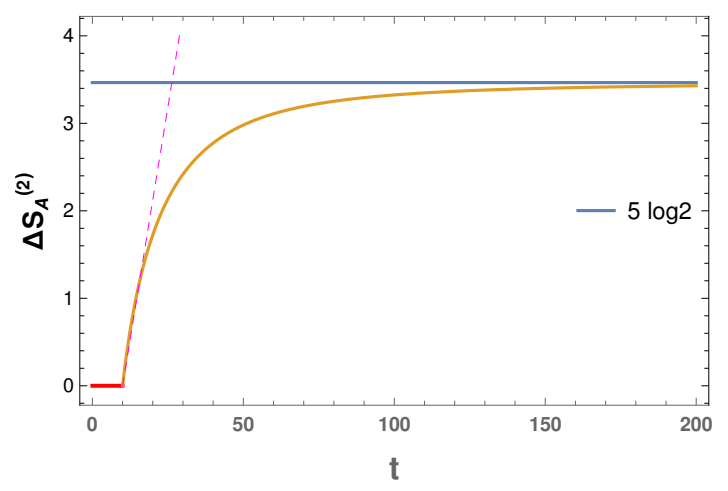

(a) $\Delta S_{A}^{(2)}$ with time for $\mathcal{I}=3$.

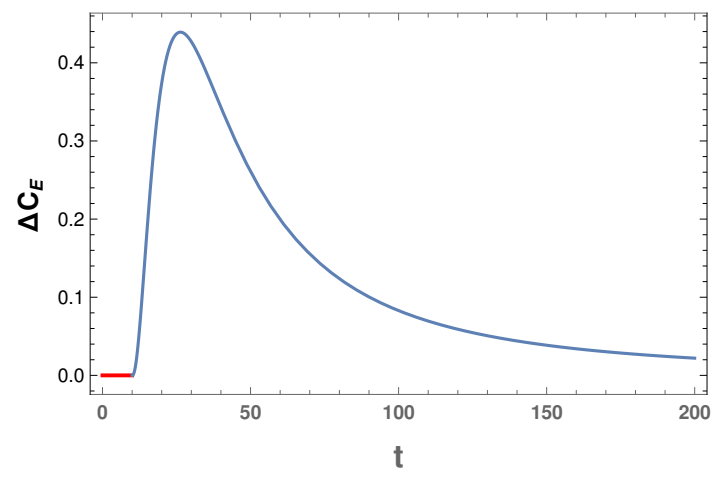

(b) $\Delta C_{E}$ with time for $\mathcal{I}=3$.

Figure 9. Plot of (a) $\Delta S_{A}^{(2)}$ and (b) $\Delta C_{E}$ for $\mathcal{I}=3$. Here we choose $L=10$. At $t \approx 26.3257$, capacity reaches a peak where the "Page transition" occurs and dies off at late times. $\Delta S_{A}^{(2)}$ saturates to $5 \log 2$. These plots are again closely similar to the entanglement entropy and capacity plots in the EoW brane model (microcanonical ensemble) [24].

value of normalized Page time. Similar to the fermionic case, we can also observe a "Page transition" here (see figure 9), and the Page time depends on $\mathcal{I}$. This is intuitively expected because, for each $\mathcal{I}$, we get a different operator. Again it concludes that the Page time can be a characteristic feature of the inserted operator at early times.

\subsection{Late time behavior and EPR interpretation}

At late times, we can directly compute the capacity of entanglement by computing Renyi entropy in terms of EPR pairs. It was shown in [34] that for $\mathcal{I}=1$ at late times, the Rényi entropies saturate to the value $\log 2$. This is because the reduced state becomes an EPR state which implies the capacity will vanish. This is intuitive as all the entanglement will be carried away by the EPR pairs, which have maximum entropy $\log 2$. For $\mathcal{I}=2$, the situation is non-trivial and interesting, where the rank of the gauge group becomes important. The Rényi entropies become (at late times) [34]

$$
\Delta S_{A}^{(m)}=\frac{1}{1-m} \log \left(2^{1-2 m}+\frac{1}{2^{m} N^{2(m-1)}}\right) .
$$

For $m \geq 2$, the $1 / N$ corrections can be neglected and at leading order, one simply finds [34]

$$
\Delta S_{A}^{(m \geq 2)} \approx \frac{2 m-1}{m-1} \log 2,
$$

while to compute the entanglement entropy at limit $m \rightarrow 1$ we need to consider the subleading term in $N$, which gives $\Delta S_{A}^{(1)}=\log (2 \sqrt{2} N)$. For the capacity, the leading order term vanishes (figure $8 \mathrm{~b}$ ). If we take into account the subleading term, we get

$$
\Delta C_{E}=\lim _{m \rightarrow 1} m^{2} \partial_{m}^{2}\left((1-m) \Delta S_{R}^{(m)}\right)=\frac{1}{4}\left[\log \left(\frac{2}{N^{2}}\right)\right]^{2} .
$$

This result is very intriguing. It shows that along with entanglement entropy, capacity is also sensitive to the internal degrees of freedom of the inserted operator in the subleading 
order. ${ }^{10}$ The difference between the entanglement entropy and the capacity is that at leading order in $N$, capacity vanishes, whereas entropy is ill-defined. Entanglement entropy is well defined only in subleading order. It will be interesting to see how the entanglement entropy and the capacity behave in different orders in large- $N$ expansion. We hope to get back to this issue in the future.

\section{Conclusion and summary}

In this paper, we have studied the time evolution of excess value of the capacity of entanglement in free, massless fermionic theory and free Yang-Mills theory in four spacetime dimensions. The result for a scalar is a particular case obtained from the Yang-Mills theory. The excess value of capacity is defined as the difference between the capacity of excited states and the capacity of the ground state. Here the excited states are obtained by applying local operators on the ground state. We found that capacity can capture the entanglement property of a given local operator similar to the series of Rényi entropies. While the Rényi entropies capture the entanglement structure at late times, capacity is an excellent probe to capture the structure early, especially when it shows a peak with a universal value at some partially entangled state. The normalized "Page time", when the capacity reaches its peak is a characteristic feature of the inserted operator. Moreover, we studied the evolution for both uncharged and charged fermionic cases. For the uncharged case, capacity dies off at late times due to the formation of EPR pairs, while for the charged case, it does not vanish at late times, instead of reaching a constant value that depends on the chemical potential. Thus, the evolution of capacity closely resembles its evolution studied in the context of the information paradox [24]. The capacity for uncharged fermion (with $\gamma=0$ ) and free Yang-Mills follows the behavior of the microcanonical ensemble of the EoW brane model. At the same time, the charged fermionic case mimics the canonical ensemble. It will be interesting to study this relation in more detail in the future.

We then studied capacity in free Yang-Mills theory. We found that capacity is well defined even after considering the large- $N$ behavior and it is well defined taking $m \rightarrow 1$ limit from Rényi entropies. This limit is ill-defined for entanglement entropy as it receives a subleading contribution. We also found a similar transition at early times here, and again it confirms capacity as an excellent probe to characterize the entanglement structure of the operator. Interestingly, capacity is sensitive to the internal degrees of freedom of the inserted operator, similar to the entanglement entropy. This contrasts with Rényi entropies that do not depend on $N$, at least in leading order.

There are many possible future directions. A straightforward problem would be to study the capacity for other fermionic operators like $\Psi^{\dagger} \Psi, \bar{\Psi} \Psi, \operatorname{tr}\left(\Psi^{\dagger} \Psi\right), \operatorname{tr}(\bar{\Psi} \Psi)$ in $d>4$ and $d=2$-dimensions. As the operators are different, the (normalized) "Page time" would be different for either case, and it would be interesting to see how it depends on dimensions. Especially in two dimensions, it would be exciting to see whether this property is valid for a conformal family similar to Rényi entropies [56]. One can also try to understand what happens for capacity in rational conformal field theories (CFTs) [37, 57]. In particular, for

\footnotetext{
${ }^{10}$ I thank Pawel Caputa for pointing this out.
} 
rational CFTs in two dimensions, the Rényi entropies and entanglement entropy exhibit a constant jump that depends on that operator's quantum dimension [35]. This implies one would expect that capacity will vanish at all times, suggesting that capacity could be a good indicator to distinguish between rational and holographic CFTs. Furthermore, it would be worth exploring the behavior of capacities with heavy operator excitation in large- $c$ CFTs $[33,58,59]$ and entanglement scrambling [60] to see the diffrence between holographic and rational CFTs. Capacity can also play a vital role in understanding some properties of symmetry resolved entropies [61-64]. It would be interesting to investigate the behavior of capacity in quench models [65-71], especially in slow and fast quench regimes in $2 d$ CFTs. This might shed light on the universal scaling behavior of capacity in different time regimes, in a similar spirit to the scaling of entropies [72]. Another exciting direction will be to explore the evolution of capacity in pure states in equilibrium [73] and connect with other information-theoretic measures like reflected entropy [74-76], complexity [77-85], and logarithmic negativity [86-88] to have a better understanding of the complete picture.

\section{Acknowledgments}

I thank Aninda Sinha, Pawel Caputa, Arpan Bhattacharyya and Aranya Bhattacharya for stimulating discussions and comments on the draft. I sincerely thank the anonymous referee for having insightful comments, which significantly improves the quality of the paper. The work is supported by University Grants Commission (UGC), Government of India.

Open Access. This article is distributed under the terms of the Creative Commons Attribution License (CC-BY 4.0), which permits any use, distribution and reproduction in any medium, provided the original author(s) and source are credited.

\section{References}

[1] A. Kitaev and J. Preskill, Topological entanglement entropy, Phys. Rev. Lett. 96 (2006) 110404 [hep-th/0510092] [INSPIRE].

[2] M. Levin and X.-G. Wen, Detecting Topological Order in a Ground State Wave Function, Phys. Rev. Lett. 96 (2006) 110405 [cond-mat/0510613] [INSPIRE].

[3] N. Laflorencie, Quantum entanglement in condensed matter systems, Phys. Rept. 646 (2016) 1 [arXiv: 1512.03388] [INSPIRE].

[4] J.M. Maldacena, The Large N limit of superconformal field theories and supergravity, Int. J. Theor. Phys. 38 (1999) 1113 [hep-th/9711200] [INSPIRE].

[5] E. Witten, Anti-de Sitter space and holography, Adv. Theor. Math. Phys. 2 (1998) 253 [hep-th/9802150] [InSPIRE].

[6] S. Ryu and T. Takayanagi, Holographic derivation of entanglement entropy from AdS/CFT, Phys. Rev. Lett. 96 (2006) 181602 [hep-th/0603001] [INSPIRE].

[7] S. Ryu and T. Takayanagi, Aspects of Holographic Entanglement Entropy, JHEP 08 (2006) 045 [hep-th/0605073] [INSPIRE].

[8] V.E. Hubeny, M. Rangamani and T. Takayanagi, A Covariant holographic entanglement entropy proposal, JHEP 07 (2007) 062 [arXiv:0705.0016] [INSPIRE]. 
[9] T. Faulkner, A. Lewkowycz and J. Maldacena, Quantum corrections to holographic entanglement entropy, JHEP 11 (2013) 074 [arXiv: 1307.2892] [INSPIRE].

[10] A. Lewkowycz and J. Maldacena, Generalized gravitational entropy, JHEP 08 (2013) 090 [arXiv: 1304.4926] [INSPIRE].

[11] S.W. Hawking, Particle Creation by Black Holes, Commun. Math. Phys. 43 (1975) 199 [Erratum ibid. 46 (1976) 206] [INSPIRE].

[12] S.W. Hawking, Breakdown of Predictability in Gravitational Collapse, Phys. Rev. D 14 (1976) 2460 [INSPIRE].

[13] A. Almheiri, T. Hartman, J. Maldacena, E. Shaghoulian and A. Tajdini, The entropy of Hawking radiation, arXiv:2006.06872 [INSPIRE].

[14] S. Raju, Lessons from the Information Paradox, arXiv:2012.05770 [INSPIRE].

[15] G. Penington, Entanglement Wedge Reconstruction and the Information Paradox, JHEP 09 (2020) 002 [arXiv: 1905.08255] [InSPIRE].

[16] A. Almheiri, R. Mahajan, J. Maldacena and Y. Zhao, The Page curve of Hawking radiation from semiclassical geometry, JHEP 03 (2020) 149 [arXiv: 1908.10996] [INSPIRE].

[17] N. Engelhardt and A.C. Wall, Quantum Extremal Surfaces: Holographic Entanglement Entropy beyond the Classical Regime, JHEP 01 (2015) 073 [arXiv:1408.3203] [INSPIRE].

[18] D.N. Page, Information in black hole radiation, Phys. Rev. Lett. 71 (1993) 3743 [hep-th/9306083] [INSPIRE].

[19] D.N. Page, Time Dependence of Hawking Radiation Entropy, JCAP 09 (2013) 028 [arXiv:1301.4995] [INSPIRE].

[20] J. De Boer, J. Järvelä and E. Keski-Vakkuri, Aspects of capacity of entanglement, Phys. Rev. D 99 (2019) 066012 [arXiv:1807.07357] [INSPIRE].

[21] Y. Nakaguchi and T. Nishioka, A holographic proof of Rényi entropic inequalities, JHEP 12 (2016) 129 [arXiv: 1606. 08443] [INSPIRE].

[22] Y.O. Nakagawa and S. Furukawa, Capacity of entanglement and the distribution of density matrix eigenvalues in gapless systems, Phys. Rev. B 96 (2017) 205108 [arXiv:1708.08924] [INSPIRE].

[23] J. de Boer, V. Godet, J. Kastikainen and E. Keski-Vakkuri, Quantum hypothesis testing in many-body systems, SciPost Phys. Core 4 (2021) 019 [arXiv:2007.11711] [inSPIRE].

[24] K. Kawabata, T. Nishioka, Y. Okuyama and K. Watanabe, Probing Hawking radiation through capacity of entanglement, JHEP 05 (2021) 062 [arXiv:2102.02425] [INSPIRE].

[25] K. Kawabata, T. Nishioka, Y. Okuyama and K. Watanabe, Replica wormholes and capacity of entanglement, arXiv:2105.08396 [INSPIRE].

[26] A. Bhattacharya, A. Chanda, S. Maulik, C. Northe and S. Roy, Topological shadows and complexity of islands in multiboundary wormholes, JHEP 02 (2021) 152 [arXiv:2010.04134] [INSPIRE].

[27] A. Bhattacharya, A. Bhattacharyya, P. Nandy and A.K. Patra, Islands and complexity of eternal black hole and radiation subsystems for a doubly holographic model, JHEP 05 (2021) 135 [arXiv:2103.15852] [inSPIRE].

[28] H. Yao and X.-L. Qi, Entanglement entropy and entanglement spectrum of the Kitaev model, Phys. Rev. Lett. 105 (2010) 080501 [arXiv: 1001.1165] [INSPIRE]. 
[29] K. Okuyama, Capacity of entanglement in random pure state, arXiv:2103.08909 [INSPIRE].

[30] M. Nozaki, T. Numasawa and T. Takayanagi, Quantum Entanglement of Local Operators in Conformal Field Theories, Phys. Rev. Lett. 112 (2014) 111602 [arXiv:1401.0539] [InSPIRE].

[31] M. Nozaki, Notes on Quantum Entanglement of Local Operators, JHEP 10 (2014) 147 [arXiv: 1405.5875] [INSPIRE].

[32] M. Nozaki, T. Numasawa and S. Matsuura, Quantum Entanglement of Fermionic Local Operators, JHEP 02 (2016) 150 [arXiv:1507.04352] [INSPIRE].

[33] P. Caputa, J. Simón, A. Štikonas and T. Takayanagi, Quantum Entanglement of Localized Excited States at Finite Temperature, JHEP 01 (2015) 102 [arXiv:1410.2287] [INSPIRE].

[34] P. Caputa, M. Nozaki and T. Takayanagi, Entanglement of local operators in large- $N$ conformal field theories, PTEP 2014 (2014) 093B06 [arXiv: 1405.5946] [INSPIRE].

[35] S. He, T. Numasawa, T. Takayanagi and K. Watanabe, Quantum dimension as entanglement entropy in two dimensional conformal field theories, Phys. Rev. D 90 (2014) 041701 [arXiv: 1403.0702] [INSPIRE].

[36] P. Caputa, M. Nozaki and T. Numasawa, Charged Entanglement Entropy of Local Operators, Phys. Rev. D 93 (2016) 105032 [arXiv:1512.08132] [INSPIRE].

[37] B. Chen, W.-Z. Guo, S. He and J.-q. Wu, Entanglement Entropy for Descendent Local Operators in $2 D$ CFTs, JHEP 10 (2015) 173 [arXiv:1507.01157] [INSPIRE].

[38] P. Caputa and M.M. Rams, Quantum dimensions from local operator excitations in the Ising model, J. Phys. A 50 (2017) 055002 [arXiv: 1609. 02428] [inSPIRE].

[39] M. Nozaki and N. Watamura, Quantum Entanglement of Locally Excited States in Maxwell Theory, JHEP 12 (2016) 069 [arXiv:1606.07076] [InSPIRE].

[40] P. Caputa, Y. Kusuki, T. Takayanagi and K. Watanabe, Evolution of Entanglement Entropy in Orbifold CFTs, J. Phys. A 50 (2017) 244001 [arXiv:1701.03110] [INSPIRE].

[41] S. He, Conformal bootstrap to Rényi entropy in $2 D$ Liouville and super-Liouville CFTs, Phys. Rev. D 99 (2019) 026005 [arXiv:1711.00624] [INSPIRE].

[42] Y. Sun and J.-R. Sun, Note on the Rényi entropy of $2 D$ perturbed fermions, Phys. Rev. D 99 (2019) 106008 [arXiv:1901.08796] [INSPIRE].

[43] J. Kudler-Flam, M. Nozaki, S. Ryu and M.T. Tan, Entanglement of Local Operators and the Butterfly Effect, arXiv:2005.14243 [INSPIRE].

[44] E. Mascot, M. Nozaki and M. Tezuka, Local Operator Entanglement in Spin Chains, arXiv:2012.14609 [INSPIRE].

[45] M. Gruber and V. Eisler, Entanglement spreading after local fermionic excitations in the XXZ chain, SciPost Phys. 10 (2021) 005 [arXiv: 2010.02708] [INSPIRE].

[46] T. Nishioka, Entanglement entropy: holography and renormalization group, Rev. Mod. Phys. 90 (2018) 035007 [arXiv: 1801.10352] [INSPIRE].

[47] E. D'Hoker, X. Dong and C.-H. Wu, An alternative method for extracting the von Neumann entropy from Rényi entropies, JHEP 01 (2021) 042 [arXiv:2008.10076] [INSPIRE].

[48] X. Dong, The Gravity Dual of Renyi Entropy, Nature Commun. 7 (2016) 12472 [arXiv: 1601.06788] [INSPIRE].

[49] G. Penington, S.H. Shenker, D. Stanford and Z. Yang, Replica wormholes and the black hole interior, arXiv: 1911.11977 [INSPIRE]. 
[50] L. Anderson, O. Parrikar and R.M. Soni, Islands with Gravitating Baths: Towards ER = $E P R$, arXiv:2103.14746 [INSPIRE].

[51] I. Akal, Y. Kusuki, N. Shiba, T. Takayanagi and Z. Wei, Entanglement Entropy in a Holographic Moving Mirror and the Page Curve, Phys. Rev. Lett. 126 (2021) 061604 [arXiv:2011.12005] [INSPIRE].

[52] P. Calabrese and J.L. Cardy, Entanglement entropy and quantum field theory, J. Stat. Mech. 0406 (2004) P06002 [hep-th/0405152] [INSPIRE].

[53] P. Calabrese and J. Cardy, Entanglement entropy and conformal field theory, J. Phys. A 42 (2009) 504005 [arXiv:0905 .4013] [INSPIRE].

[54] A. Belin, L.-Y. Hung, A. Maloney, S. Matsuura, R.C. Myers and T. Sierens, Holographic Charged Renyi Entropies, JHEP 12 (2013) 059 [arXiv: 1310.4180] [INSPIRE].

[55] A. Belin, L.-Y. Hung, A. Maloney and S. Matsuura, Charged Renyi entropies and holographic superconductors, JHEP 01 (2015) 059 [arXiv: 1407.5630] [INSPIRE].

[56] P. Caputa and A. Veliz-Osorio, Entanglement constant for conformal families, Phys. Rev. D 92 (2015) 065010 [arXiv: 1507.00582] [INSPIRE].

[57] T. Numasawa, Scattering effect on entanglement propagation in RCFTs, JHEP 12 (2016) 061 [arXiv: 1610.06181] [INSPIRE].

[58] C.T. Asplund, A. Bernamonti, F. Galli and T. Hartman, Holographic Entanglement Entropy from 2d CFT: Heavy States and Local Quenches, JHEP 02 (2015) 171 [arXiv:1410.1392] [INSPIRE].

[59] P. Caputa, J. Simón, A. Štikonas, T. Takayanagi and K. Watanabe, Scrambling time from local perturbations of the eternal BTZ black hole, JHEP 08 (2015) 011 [arXiv:1503.08161] [INSPIRE].

[60] C.T. Asplund, A. Bernamonti, F. Galli and T. Hartman, Entanglement Scrambling in $2 d$ Conformal Field Theory, JHEP 09 (2015) 110 [arXiv:1506.03772] [INSPIRE].

[61] R. Bonsignori, P. Ruggiero and P. Calabrese, Symmetry resolved entanglement in free fermionic systems, J. Phys. A 52 (2019) 475302 [arXiv:1907.02084] [InSPIRE].

[62] M.T. Tan and S. Ryu, Particle number fluctuations, Rényi entropy, and symmetry-resolved entanglement entropy in a two-dimensional Fermi gas from multidimensional bosonization, Phys. Rev. B 101 (2020) 235169 [arXiv:1911.01451] [INSPIRE].

[63] L. Capizzi, P. Ruggiero and P. Calabrese, Symmetry resolved entanglement entropy of excited states in a CFT, J. Stat. Mech. 2007 (2020) 073101 [arXiv:2003.04670] [INSPIRE].

[64] S. Murciano, G. Di Giulio and P. Calabrese, Entanglement and symmetry resolution in two dimensional free quantum field theories, JHEP 08 (2020) 073 [arXiv:2006.09069] [INSPIRE].

[65] J.R. David, S. Khetrapal and S.P. Kumar, Universal corrections to entanglement entropy of local quantum quenches, JHEP 08 (2016) 127 [arXiv: 1605.05987] [INSPIRE].

[66] Y. Kusuki and T. Takayanagi, Renyi entropy for local quenches in $2 D$ CFT from numerical conformal blocks, JHEP 01 (2018) 115 [arXiv:1711.09913] [INSPIRE].

[67] P. Caputa, T. Numasawa, T. Shimaji, T. Takayanagi and Z. Wei, Double Local Quenches in $2 D$ CFTs and Gravitational Force, JHEP 09 (2019) 018 [arXiv: 1905.08265] [INSPIRE].

[68] A. Bhattacharyya, T. Takayanagi and K. Umemoto, Universal Local Operator Quenches and Entanglement Entropy, JHEP 11 (2019) 107 [arXiv:1909.04680] [INSPIRE]. 
[69] J. Zhang and P. Calabrese, Subsystem distance after a local operator quench, JHEP 02 (2020) 056 [arXiv : 1911.04797] [INSPIRE].

[70] Y. Kusuki and M. Miyaji, Entanglement Entropy after Double Excitation as an Interaction Measure, Phys. Rev. Lett. 124 (2020) 061601 [arXiv:1908.03351] [INSPIRE].

[71] G. Parez, R. Bonsignori and P. Calabrese, Quasiparticle dynamics of symmetry-resolved entanglement after a quench: Examples of conformal field theories and free fermions, Phys. Rev. B 103 (2021) L041104 [arXiv: 2010. 09794] [INSPIRE].

[72] P. Caputa, S.R. Das, M. Nozaki and A. Tomiya, Quantum Quench and Scaling of Entanglement Entropy, Phys. Lett. B $\mathbf{7 7 2}$ (2017) 53 [arXiv:1702. 04359] [INSPIRE].

[73] H. Liu and S. Vardhan, Entanglement entropies of equilibrated pure states in quantum many-body systems and gravity, P. R. X. Quantum. 2 (2021) 010344 [arXiv:2008.01089] [INSPIRE].

[74] S. Dutta and T. Faulkner, A canonical purification for the entanglement wedge cross-section, JHEP 03 (2021) 178 [arXiv: 1905.00577] [INSPIRE].

[75] P. Bueno and H. Casini, Reflected entropy, symmetries and free fermions, JHEP 05 (2020) 103 [arXiv: 2003.09546] [INSPIRE].

[76] P. Bueno and H. Casini, Reflected entropy for free scalars, JHEP 11 (2020) 148 [arXiv: 2008.11373] [INSPIRE].

[77] R. Jefferson and R.C. Myers, Circuit complexity in quantum field theory, JHEP 10 (2017) 107 [arXiv: 1707.08570] [INSPIRE].

[78] R. Khan, C. Krishnan and S. Sharma, Circuit Complexity in Fermionic Field Theory, Phys. Rev. D 98 (2018) 126001 [arXiv:1801.07620] [InSPIRE].

[79] A. Bhattacharyya, A. Shekar and A. Sinha, Circuit complexity in interacting QFTs and RG flows, JHEP 10 (2018) 140 [arXiv:1808.03105] [InSPIRE].

[80] A. Bhattacharyya, P. Nandy and A. Sinha, Renormalized Circuit Complexity, Phys. Rev. Lett. 124 (2020) 101602 [arXiv: 1907.08223] [INSPIRE].

[81] V. Balasubramanian, M. Decross, A. Kar and O. Parrikar, Quantum Complexity of Time Evolution with Chaotic Hamiltonians, JHEP 01 (2020) 134 [arXiv: 1905. 05765] [INSPIRE].

[82] P. Caputa, N. Kundu, M. Miyaji, T. Takayanagi and K. Watanabe, Anti-de Sitter Space from Optimization of Path Integrals in Conformal Field Theories, Phys. Rev. Lett. 119 (2017) 071602 [arXiv: 1703.00456] [inSPIRE].

[83] P. Caputa, N. Kundu, M. Miyaji, T. Takayanagi and K. Watanabe, Liouville Action as Path-Integral Complexity: From Continuous Tensor Networks to AdS/CFT, JHEP 11 (2017) 097 [arXiv: 1706 . 07056] [inSPIRE].

[84] A. Bhattacharyya, P. Caputa, S.R. Das, N. Kundu, M. Miyaji and T. Takayanagi, Path-Integral Complexity for Perturbed CFTs, JHEP 07 (2018) 086 [arXiv:1804.01999] [INSPIRE].

[85] S. Chapman, M.P. Heller, H. Marrochio and F. Pastawski, Toward a Definition of Complexity for Quantum Field Theory States, Phys. Rev. Lett. 120 (2018) 121602 [arXiv: 1707.08582] [INSPIRE].

[86] P. Calabrese, J. Cardy and E. Tonni, Entanglement negativity in quantum field theory, Phys. Rev. Lett. 109 (2012) 130502 [arXiv:1206. 3092] [INSPIRE]. 
[87] P. Calabrese, J. Cardy and E. Tonni, Entanglement negativity in extended systems: A field theoretical approach, J. Stat. Mech. 1302 (2013) P02008 [arXiv: 1210. 5359] [INSPIRE].

[88] J. Kudler-Flam, Y. Kusuki and S. Ryu, The quasi-particle picture and its breakdown after local quenches: mutual information, negativity, and reflected entropy, JHEP 03 (2021) 146 [arXiv:2008.11266] [INSPIRE]. 